\title{
Magnetic Properties and Particle Flowability of Neodymium Magnetic Granules
}

\author{
Yasushi Enokido ${ }^{1, *}$, Fumitaka Baba ${ }^{2}$ and Yoshitaka Kitamoto ${ }^{3}$ \\ ${ }^{1}$ Materials Research Center, Technology and Intellectual Property HQ, TDK Corporation, Narita 286-8588, Japan \\ ${ }^{2}$ Magnet Products Business Group, TDK Corporation, Narita 286-8588, Japan \\ ${ }^{3}$ Department of Materials Science and Engineering, Tokyo Institute of Technology, Yokohama 226-8503, Japan
}

In this study, sintered Nd-Fe-B magnets with high residual flux densities and magnetic coercivities were manufactured by filling a die cavity with granulated $\mathrm{Nd}-\mathrm{Fe}-\mathrm{B}$ magnetic powder during pressing. In the binder-bound granules prepared by a spray drying method, the bonding force between the primary particles was relatively strong. However, the magnets obtained from these granules could not achieve high residual magnetic flux densities because it was difficult to align each particle in these granules along the magnetization easy axis through magnetic field pressing. Therefore, a weak liquid bridge force was utilized for particle binding. After preparing the liquid-bound granules containing 0.25 mass $\%$ terpineol, which does not evaporate easily at room temperature $(\sim 293 \mathrm{~K})$ but undergoes debinding on baking, the angle of repose of the magnetic powder (representing its flowability index) decreased from $63^{\circ}$ to $44.2^{\circ}$, while the deterioration of its magnetic properties was considerably suppressed. As a result, the residual magnetic flux density of the sintered magnet was equal to $99.3 \%$ of the value obtained for the non-granulated powder. In addition, the liquid-bound granules exhibited high filling rates and low weight variations, demonstrating their applicability for mass production. [doi:10.2320/matertrans.MT-M2021017]

(Received February 2, 2021; Accepted April 5, 2021; Published June 25, 2021)

Keywords: neodymium magnet, liquid-bound granule, flowability, angle of repose, magnetization

\section{Introduction}

Sagawa et al. first reported neodymium-iron-boron $(\mathrm{Nd}-$ Fe-B) sintered magnets in $1984 .^{1,2)}$ Since then, these magnets have been used worldwide because of their excellent magnetic properties, including high residual magnetic flux density and coercivity. Owing to the high prices of rare-earth elements, ${ }^{3)}$ material costs constitute large fractions of production costs; hence, the effective use of starting materials is critical for manufacturing magnets.

Magnets are generally used in close contact with soft magnetic materials, and the gap between these two components during assembly should be minimized to reduce the influence of the demagnetizing field. Therefore, the majority of magnetic products have polished surfaces. In many cases, magnetic products with complex shapes, such as the arc-shaped magnets used for surface permanent magnet motors, are obtained from rectangular magnets by grinding them to the desired dimensions. Such grinding and polishing steps significantly reduce the material yield of magnetic products, thereby wasting precious rare-earth resources. To minimize the amount of grinding and polishing waste, several net-shaping techniques have been proposed, ${ }^{4}$ such as wet, ${ }^{5)}$ hot deformation, ${ }^{6}$ ) metal injection molding, ${ }^{7)}$ and additive manufacturing. ${ }^{8)}$ However, these techniques are not suitable for mass production because of the high processing costs.

Therefore, to align the magnetization directions of various domains during the regular magnet manufacturing procedure, net-shape pressing must be performed uniaxially under an external magnetic field. Transverse pressing, in which the magnetic field is applied perpendicularly to the pressing force, produces magnets with higher residual flux densities than those obtained via axial pressing. The majority of the shapes utilized for fabricating high-performance magnets have shorter dimensions along the direction of the magnetic field; hence, the transverse pressing method is widely used

*Corresponding author, E-mail: Yasushi.Enokido@tdk.com for mass production, despite the limited number of processable shapes. Because the utilized die cavity has a relatively narrow opening and large depth, increasing the flowability of the magnetic powder is extremely important for meeting the requirements of high-performance magnetic products. ${ }^{9,10)}$ It also facilitates the filling of narrow cavities, increases the filling rate, and decreases the weight variations of the molded product. Note that it is extremely difficult to uniformly fill multiple cavities with the same volume of the magnetic powder characterized by low flowability. Furthermore, high pressure is applied only to a small number of cavities, which ultimately leads to mold breakage.

Binder-bound granules prepared by a spray drying method are widely used to manufacture the ceramic parts of electronic components. Such granules represent secondary particles (aggregates of primary particles) with excellent flowability properties due to their relatively large sizes ${ }^{11)}$ and round shapes. However, because it is difficult to align each primary particle in a binder-bound granule along the easy axis direction, the granule pressing technique cannot be practically used for magnet manufacture. To apply the spray drying method, the bonding strength of each particle in the granule should be lower than the rotational force exerted by the magnetic field; otherwise, a sufficient degree of orientation cannot be achieved for an anisotropic magnet. In the late 1990s, Yamashita et al. performed multiple studies on the granulation of $\mathrm{Nd}-\mathrm{Fe}-\mathrm{B}$ powder ${ }^{12-17)}$ to address these issues. The granules prepared from a slurry containing polyvinyl alcohol (PVA), water, and magnetic powder exhibited high flowability, while the magnets obtained by pressing and sintering these granules possessed a relatively low residual magnetic flux density, corresponding to $90-95 \%$ of the value obtained for the non-granulated powder. ${ }^{14)}$ Owing to the increasing market demand for high-performance magnets, it is necessary to minimize the deterioration of magnetic properties caused by the use of granules in their fabrication. In this study, we evaluate a novel method for achieving high residual flux density, magnetic coercivity, and 
flowability of the Nd-Fe-B powder utilized for large-scale magnet production.

\section{Experimental Procedure}

\subsection{Preparation of primary magnetic particles}

Two different alloys with the compositions 12.1 at $\% \mathrm{Nd}-$ 2.4 at\% Dy-0.5 at\% Co-1.0 at\% B-M-bal. Fe ( $\mathrm{M}=\mathrm{Cu}, \mathrm{Al})$ and 12.6 at $\%$ Nd-1.8 at\% Dy-0.5 at\% Co-1.0 at\% B-M-bal. $\mathrm{Fe}(\mathrm{M}=\mathrm{Cu}, \mathrm{Al})$ were produced by a strip casting method. Powders made from the two alloys are referred to as Powder A and Powder B, respectively. These alloys possessed different magnetization and coercivity properties due to the different contents of $\mathrm{Nd}$ and Dy. The raw alloys were coarsely pulverized by a hydrogen decrepitation method followed by fine pulverization via jet milling to obtain fine powders with an average particle diameter of $4.7 \mu \mathrm{m}$. The pulverization process was performed in an anaerobic atmosphere with an oxygen content of $3000 \mathrm{ppm}$.

\subsection{Preparation of magnetic granules \\ 2.2.1 Binder-bound granules}

A slurry of magnetic particles was prepared from a primary magnetic powder, an organic binder, and a solvent without a dispersant. The primary particles obtained from one of the two above-mentioned alloys were used as the magnetic powder. PVA (PVA 217, Kuraray Co., Ltd.) with a saponification degree of $87-89 \%$ and polymerization degree of 1700, and polyvinyl butyral (PVB BH-3, Sekisui chemical co., Ltd.) with a polymerization degree of 1700 were utilized as the organic binders. Deionized water and ethanol were used as the solvents. First, an organic binder was completely dissolved in a solvent followed by the addition of the magnetic powder and mixing by an agitator. Powder concentration in the slurry was $15 \mathrm{vol} \%$.

The binder-bound granules were produced by spraying the slurry using a rotary-disk-type spray drier (CL-12, Ohkawara Kakohki, Co., Ltd.) under a nitrogen atmosphere to avoid high-temperature oxidation of the magnetic powder. The rotational speed of the rotary disk was $14000 \mathrm{rpm}$. The inlet and outlet temperatures of the drying chamber were $343-$ $373 \mathrm{~K}$ and $323-348 \mathrm{~K}$, respectively. A flowchart of the preparation of a sintered magnet using binder-bound granules is shown in Fig. 1(a).

\subsubsection{Liquid-bound granules}

A tumbling granulator (RMO-2H, Dalton Co., Ltd.) was used to prepare the liquid-bound granules. The primary particles were placed inside the granulator chamber under a nitrogen gas flow. Chopper rotation speed was $4700 \mathrm{rpm}$. The binding liquid was sprayed after the chamber was completely filled with nitrogen, and the chopper rotation speed was changed to $2300 \mathrm{rpm}$ for $1 \mathrm{~min}$ for leveling the granule size. A flowchart of the preparation of a sintered magnet using liquid-bound granules is shown in Fig. 1(b).

\subsection{Characterization techniques}

Scanning electron microscopy (SEM) and optical microscopy were used for observing the granule morphology. A static angle of repose ${ }^{18}$ ) was measured as the powder flowability indicator by a ABD powder peculiarity measuring (a)

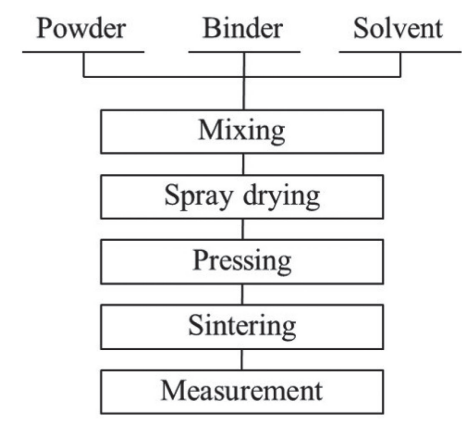

(b)

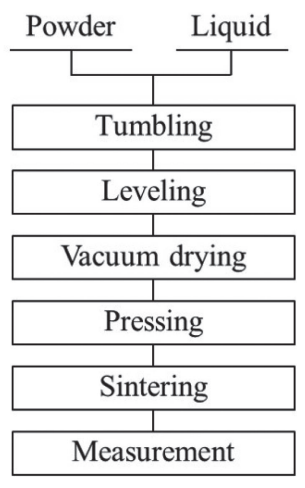

Fig. 1 Flowcharts of the preparation of sintered magnets using (a) binderbound granules and (b) liquid-bound granules.

instrument (Tsutsui Scientific Instruments Co., Ltd.). Using a vibrating mesh, the granules were dropped from a funnel onto a sample table with a diameter of $60 \mathrm{~mm}$ to form a conical pile, and thereafter, the angle between the pile peak and the table surface was determined. This process was repeated three times, and the average value of the measured angles of repose was calculated (generally, a low angle of repose indicates a higher powder flowability).

Primary or secondary particles (granules) were placed in a transverse magnetic field of $1600 \mathrm{kA} / \mathrm{m}$ to produce compacts by uniaxial pressing under a pressure of $137 \mathrm{MPa}$. To prepare binder-bound granules, the debinding process of heating at $400^{\circ} \mathrm{C}$ for $1 \mathrm{~h}$ in a hydrogen atmosphere was performed prior to the sintering procedure. The obtained compacts were sintered in vacuum $\left(10^{-3} \mathrm{~Pa}\right)$ for $4 \mathrm{~h}$ in a temperature range of $1333-1353 \mathrm{~K}$ and then annealed for $1 \mathrm{~h}$ at $1123 \mathrm{~K}$ followed by annealing for $1 \mathrm{~h}$ at $833 \mathrm{~K}$. The compacting process was conducted in air. The magnetic properties of the resulting specimens were evaluated using a B-H curve tracer (Tamakawa Co., Ltd.). The magnetic properties of the sintered bodies prepared by pressing and sintering the primary powders of Powder A and Powder B exhibit a residual flux density of approximately $1260 \mathrm{mT}$ and a coercivity of $2030 \mathrm{kA} / \mathrm{m}$, and a residual flux density of $1300 \mathrm{mT}$ and a coercivity of $1790 \mathrm{kA} / \mathrm{m}$, respectively.

\section{Results and Discussions}

\subsection{Binder-bound granules}

The binder-bound granules were prepared by the widely used spray drying method. In this procedure, ensuring proper solvent selection is critical because the magnetic properties of the neodymium magnetic powder (especially its coercivity) may deteriorate due to oxidation. Therefore, $3 \mathrm{~g}$ of Powder A was added to $8 \mathrm{~cm}^{3}$ of various solvents for $8 \mathrm{~h}$; the resulting mixtures were kept uncovered and vacuum dried at a room temperature of $293 \mathrm{~K}$. The oxygen contents in the solventtreated and non-treated powders are listed in Table 1. Significant powder oxidation was observed after the treatment with deionized water. ${ }^{12,13)}$ Because toluene is less compatible with water than with ethanol, a lower degree of oxidation was expected for the toluene-treated powder. However, the degrees of oxidation obtained for the magnetic 
Table 1 Oxygen contents in the magnetic powders immersed in various solvents.

\begin{tabular}{ccccc}
\hline & Non-immersed & Deionized water & Ethanol & Toluene \\
\hline \hline Oxygen (ppm) & 3960 & 13130 & 5210 & 5160 \\
\hline
\end{tabular}
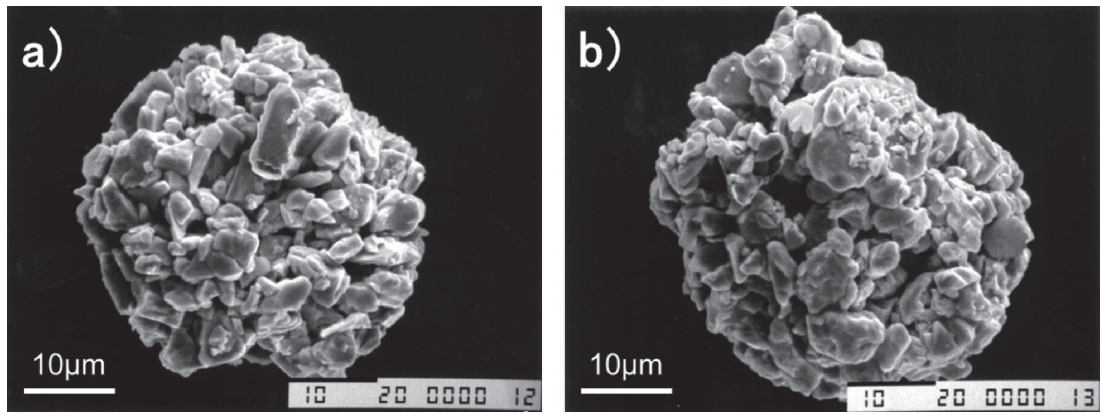

Fig. 2 SEM images of (a) PVA and (b) PVB granules.

Table 2 Angles of repose of the spray dried granules and magnetic properties of the sintered magnets prepared from these granules.

\begin{tabular}{lccc}
\hline & Angle of repose $\left(^{\circ}\right)$ & $B r(\%)$ & $H_{c J}(\%)$ \\
\hline \hline Non-granulated & 63 & 100 & 100 \\
PVA+DIW & 36.7 & 49.8 & 7.7 \\
PVB+Ethanol & 30.0 & 64.1 & 100.6 \\
\hline
\end{tabular}

powders soaked in toluene and ethanol were almost identical. Hence, ethanol was used as the organic solvent for the binder due to its lower cost.

Two types of the spray dried granules with a binder content of 0.8 mass\% were prepared with using Powder A: PVA in deionized water and PVB in ethanol. SEM images of these granules are shown in Fig. 2. Their surfaces are not smooth due to the large size of the primary particles; however, no significant differences between the shapes of the two granules are observed. The average diameters of both granules are approximately $40 \mu \mathrm{m}$.

Despite the existence of several powder flowability indicators, including the Carr flowability index, ${ }^{18)}$ the angle of repose is the most suitable metric for estimating the flowability range of the primary and secondary particles in a magnet. The angles of repose of the spray dried granules and magnetic properties of the sintered magnets prepared from these granules are listed in Table 2 (here, the nongranulated powder is used as the reference). Both granule types have much smaller angles of repose than that of the non-granulated powder, indicating higher flowabilities; however, their magnetic properties, namely residual flux density and coercivity, are significantly degraded.

The PVA granules were not sufficiently densified and exhibited large deformation after sintering; as a result, their sintered density could not be measured. In contrast, the PVB granules were densified to a value exceeding $95 \%$ of their theoretical density. The particle oxidization in the PVA granules reduced the content of the R-rich phase and

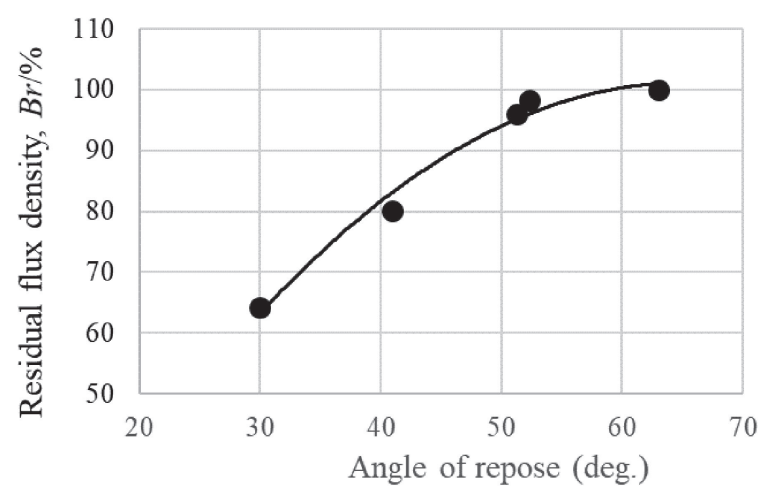

Fig. 3 Relationship between the angle of repose and the relative residual flux density of the prepared granules.

prevented sintering of the liquid phase; ${ }^{19)}$ it can also explain their low magnetic coercivity. ${ }^{20,21)}$ Both granule types possessed extremely low values of the residual flux density, which was proportional to the relative density (ratio of actual to theoretical density) and degree of orientation. The PVB granules with high sintered density also exhibited a low residual magnetic flux density, which was likely caused by the low degree of orientation because the particle bonding by the organic binder prevented the rotation of the primary particles against the applied magnetic field during pressing. ${ }^{22)}$

To increase the granule residual flux density, the binder content in the PVB granules was decreased to weaken the binding strength of each particle. The obtained relationship between the angle of repose and the relative residual flux density of the granules with PVB contents of 0.0 (nongranulated), $0.1,0.3,0.5$, and 0.8 mass $\%$ is presented in Fig. 3, while the SEM images of these granules are displayed in Fig. 4. According to Fig. 3, the angle of repose decreases with increasing binder content, and the SEM observations show that the primary and secondary particles coexist in the granules with low PVB contents. From these results, it has been concluded that some granules collapse during the spraydrying process and that it is difficult to maintain the granular state at an organic binder concentration of 0.1 mass $\%$ or less. 

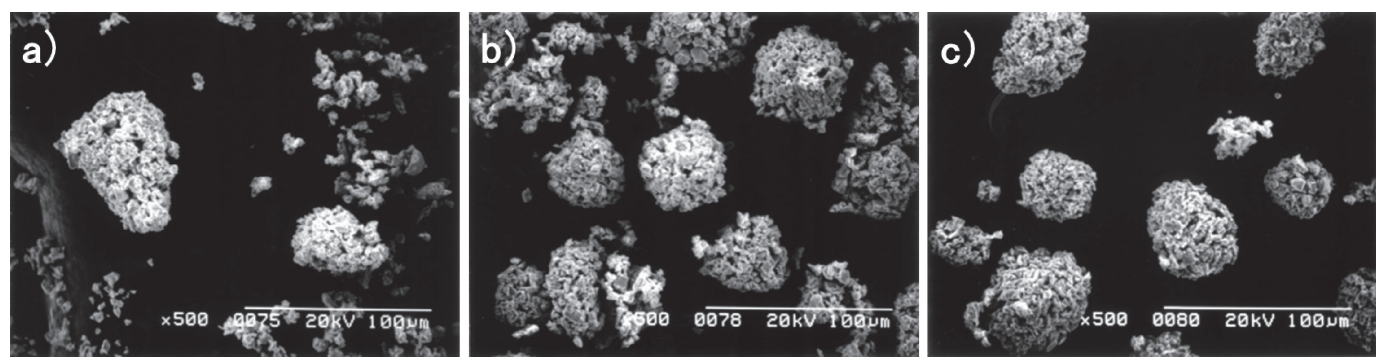

Fig. 4 SEM images of the PVB granules with PVB contents of (a) 0.10 mass $\%$, (b) 0.30 mass $\%$, and (c) 0.50 mass $\%$.

Table 3 Vapor pressures of the utilized liquids at $1793 \mathrm{~K}$, concentrations of the residual oxygen and carbon, and angles of repose of granules prepared from the corresponding liquids.

\begin{tabular}{|c|c|c|c|c|}
\hline & $\begin{array}{c}\text { Vapor pressure } \\
(\mathrm{mmHg})\end{array}$ & $\begin{array}{c}\mathrm{O} \\
(\mathrm{ppm})\end{array}$ & $\begin{array}{c}\mathrm{C} \\
(\mathrm{ppm})\end{array}$ & $\begin{array}{c}\text { Angle of repose } \\
\left({ }^{\circ}\right)\end{array}$ \\
\hline Non-granulated & - & 4360 & 790 & 63 \\
\hline Ethanol & 44 & 5030 & 850 & 54 \\
\hline Toluene & 22 & 4690 & 880 & 54 \\
\hline Terpineol & 0.04 & 10940 & 2730 & 43 \\
\hline
\end{tabular}

The binding force of the organic binder cannot withstand external forces (such as the centrifugal and impact ones), which are applied to the granules during the spray drying process. The primary particles may easily rotate during the magnetic field pressing to achieve a higher residual flux density; however, the rotation of the secondary particles is hindered. Therefore, the residual magnetic flux density increased as the fraction of the primary particles increased. To achieve the optimal residual flux density and flowability of the granules, the latter should be granulated by the relatively weak external forces. Furthermore, because the debinding heat treatment of the organic binder conducted under the hydrogen atmosphere considerably increases the production cost, granules should be preferably densified under normal sintering conditions.

\subsection{Liquid-bound granules}

Because the primary particles must be bound to each other by a bonding force that is weaker than that of the organic binder, a liquid bridge force was used for this purpose. ${ }^{23,24)}$ Three liquids (ethanol, toluene, and terpineol) were selected based on their vapor pressures, which allowed them to easily debind during the sintering process; otherwise, the produced residual carbon might decrease the granule coercivity. The primary particles $(100 \mathrm{~g})$ of Powder A and specific amounts of the liquid were mixed manually using a mortar to form granules. For uniform mixing, 12.5 mass $\%$ of ethanol, 12.5 mass $\%$ of toluene, and 6.25 mass $\%$ of terpineol were added to the magnetic powder. Terpineol undergoes limited volatization during mixing; therefore, its content was much lower than those of ethanol and toluene. During mixing, the primary particles adsorbed the liquid and formed lumps through tumbling agglomeration, ${ }^{25,26)}$ which were subsequently granulated. The obtained granules were sieved through $300 \mu \mathrm{m}$ and $180 \mu \mathrm{m}$ mesh sieves to select granules with intermediate sizes. Table 3 lists the vapor pressures of all the liquids at $293 \mathrm{~K}$, concentrations of the residual oxygen and carbon elements, and angles of repose of the granules prepared from different liquids (the non-granulated powder was used as the reference).

The granules prepared from ethanol and toluene contained low contents of carbon and oxygen residues, which likely resulted from the volatilization of the organic liquids. Further, these granules had smaller angles of repose than the nongranulated powder. In contrast, the granules prepared with terpineol had larger amounts of residual carbon and oxygen and a much smaller angle of repose than the non-granulated powder.

The granules were pressed in the presence of a magnetic field and sintered, after which the concentrations of the residual oxygen and carbon were measured, and the magnetic properties of the granules were determined. No special debinding procedure, such as heating under a hydrogen atmosphere, was performed for the spray dried granules. The concentrations of residual elements and magnetic properties of the granules are listed in Table 4. The contents of the residual oxygen and carbon in each specimen were relatively low, indicating effective debinding of these species in the sintering process. The terpineol granules possessed a slightly higher carbon content, which reduced the granule and residual flux densities; however, no apparent decrease in the magnetic coercivity was observed. Hence, the liquidbound granules prepared using terpineol were most suitable for magnet fabrication in terms of flowability and magnetic properties.

Furthermore, to scale up the production of liquid-bound granules, the tumbling granulator specified in section 2.2 was utilized (the spray drying method could not be applied to the 
Table 4 Residual oxygen and carbon contents, and magnetic properties of sintered magnets obtained from the granules treated with various liquids.

\begin{tabular}{lccccc}
\hline Mixed liquid & $\mathrm{O}(\mathrm{ppm})$ & $\mathrm{C}(\mathrm{ppm})$ & $\begin{array}{c}\text { Density } \\
\left(\mathrm{Mg} / \mathrm{m}^{3}\right)\end{array}$ & $B_{r}(\%)$ & $H_{c J}(\%)$ \\
\hline \hline Non-granulated & 4360 & 580 & 7.59 & 100 & 100 \\
Ethanol & 4530 & 640 & 7.59 & 99.3 & 99.2 \\
Toluene & 4760 & 610 & 7.59 & 99.9 & 99.8 \\
Terpineol & 4680 & 690 & 7.56 & 97.5 & 99.7 \\
\hline
\end{tabular}

liquid-bound granules because it would disintegrate them due to the weakness of the liquid bridge force). This apparatus contained a cylindrical chamber oriented in the horizontal direction, a mixing blade, two choppers, and a two-fluid nozzle inside the chamber. The powder placed in the chamber was scraped with the mixing blade and then dispersed and shredded with the two choppers to ensure uniform mixing with a low degree of segregation. The liquid was sprayed through the two-fluid nozzle using nitrogen gas. When its amount was too small, the liquid and the powder could not be uniformly mixed. Even using the granulator, it was still difficult to homogeneously mix the powder and the granulate with a terpineol content less than 6.25 mass $\%(\approx 35 \mathrm{vol} \%)$ because of its inability to reach the pendular state. ${ }^{26)}$ However, the terpineol concentration of 6.25 mass $\%$ was too high to obtain a high residual magnetic flux density. To achieve both the uniform mixing and high residual flux density, the tumbling granulation process was performed by jetting a mixture of two liquids with different vapor pressures. Thereafter, only the liquid with the high vapor pressure was evaporated and removed from the liquid-bound granule in a vacuum dryer. By applying this method, the amount of terpineol required for achieving good magnetic properties of the liquid-bound granules can be accurately controlled while adding the required liquid amount.

The liquid-bound granules were prepared in the granulator by adding 2.0 mass $\%$ of terpineol and 6.75 mass $\%$ of ethanol to the magnetic powder of Powder $\mathrm{A}$ at a jetting rate of 50 $\mathrm{g} / \mathrm{min}$. The vapor pressures of terpineol and ethanol differed by three orders of magnitude, and therefore, were suitable for the selective removal of the liquids through vacuum evaporation. The granules larger than $425 \mu \mathrm{m}$ were overcut using an automatic sieve; however, an undercutting procedure was not performed. Moreover, only ethanol was removed by vacuum drying and collected through a cold trap for further reuse in manufacturing liquid-bound granules. The angle of repose and photograph of the sieved liquidbound granules obtained using the non-granulated powder as the reference are shown in Fig. 5. Thus, liquid-bound granules were successfully granulated by utilizing the tumbling granulator and the automatic sieving machine as well as by manual mixing in a mortar.

The granule size distributions obtained before and after sieving are shown in Fig. 6. The sieved liquid-bound granules clearly exhibit a lower angle of repose than the

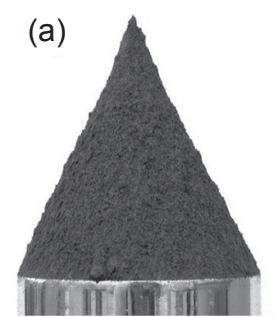

(b)

(c)

Fig. 5 Angles of repose of the (a) original magnetic powder and (b) liquidbound granule. (c) An optical image of the liquid-bound granule containing 2.0 mass $\%$ of terpineol.

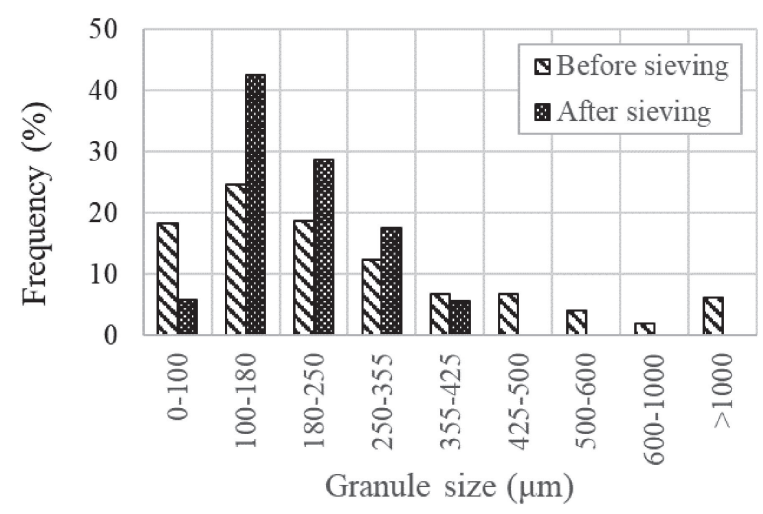

Fig. 6 Granule size distributions obtained before and after sieving.

non-sieved granules. During automatic sieving, more intense tumbling was applied to the granules because of the vibration of the sieve; as a result, fine granules with sizes $<180 \mu \mathrm{m}$ merged with the coarser granules, thus improving the size distribution. The angles of repose of the granules measured before and after sieving as well as the magnetic properties of the sintered magnets obtained from these granules are listed in Table 5. These results indicate that the angle of repose of the granules evidently decreased after sieving; however, a small reduction in the residual flux density was observed for the sintered magnets fabricated from these granules. This phenomenon was likely caused by the decrease in the number of the primary magnetic particles, which were easily rotated during magnetic field pressing. Furthermore, because the weight loss during sintering was approximately 2 mass $\%$, only ethanol species were effectively removed by the vacuum evaporation process. 
Table 5 Angles of repose of granules obtained before and after sieving, and magnetic properties of the sintered magnets prepared from these granules.

\begin{tabular}{lccc}
\hline & Angle of repose $\left(^{\circ}\right)$ & $B_{r}(\%)$ & $H_{c J}(\%)$ \\
\hline \hline Non-granulated & 63 & 100 & 100 \\
Before sieving & 52.3 & 98.6 & 99.4 \\
After sieving & 44.8 & 97.8 & 98.2 \\
\hline
\end{tabular}

Table 6 Angles of repose of the terpineol-bound granules measured at different terpineol concentrations, and magnetic properties of the sintered magnets prepared from these granules.

\begin{tabular}{lccc}
\hline $\begin{array}{l}\text { Terpineol } \\
(\mathrm{mass} \%)\end{array}$ & Angle of repose $\left({ }^{\circ}\right)$ & $B_{r}(\%)$ & $H_{c J}(\%)$ \\
\hline \hline Non-granulated & 63 & 100 & 100 \\
0.125 & 44.2 & 99.6 & 98.5 \\
0.25 & 44.2 & 99.3 & 99.3 \\
0.5 & 42.7 & 98.8 & 99.7 \\
1.0 & 42.2 & 98.6 & 99.0 \\
\hline
\end{tabular}

To increase the residual flux density of the sintered magnets, the terpinol concentration was decreased. A high jetting rate of the liquid was used $(70 \mathrm{~g} / \mathrm{min})$ according to the results of previous studies, because for a higher jetting rate of the liquid, larger granule diameters were obtained, which increased the particle flowability. Automatic sieving was performed to overcut the granules with sizes larger than $450 \mu \mathrm{m}$. The angles of repose of the liquid-bound granules with using Powder B measured at different terpineol concentrations and the magnetic properties of the sintered magnets fabricated from these granules are listed in Table 6. Although the flowability of the granules and their residual flux density exhibit a reciprocal relationship, a higher residual magnetic flux density was obtained at a smaller angle of repose than that of the binder-bound granules. In the specified terpineol addition range, no changes in the coercivity of the sintered magnets were observed. At a terpineol concentration of approximately 0.25 mass $\%(\approx 2.0 \mathrm{vol} \%)$, both the high flowability and residual flux density (compared with those of the spray dried granules) were achieved. A $0.7 \%$ reduction in the residual magnetic flux density corresponds to $9 \mathrm{mT}$, which represents the grade of magnetic properties employed for the non-granulated material.

\subsection{Flowability evaluation on a mass production line}

Using a mass production uniaxial pressing machine, weight variations of the pressed powder fraction were investigated. During the mass production process, the powder filling rate must be sufficiently high, while the weight variations of the filled part should be small. Filling a constant volume requires a constant bulk density of the powder. The utilized die contained two cavities with opening dimensions of $5 \mathrm{~mm} \times 14 \mathrm{~mm}$ and filling depth of approximately $60 \mathrm{~mm}$. A powder feeder box was moved back and forth above the cavity, causing the powder to fall naturally onto its top. The time required for powder filling was determined, and pressing was performed 50 times under the same conditions. The variation $\sigma$ and average avg of the weight for the two compacts in the two cavities were obtained and the ratio of the variation to the average $\sigma / \mathrm{avg}$ was calculated every pressing. The ratio $\sigma /$ avg was averaged for 50 pressings to obtain the intra-pressure weight variation in a single pressing. The standard deviation and mean of the average weight avg for the two compacts in one pressing were calculated from 50 pressing and the ratio of the standard deviation to the mean was calculated to evaluate the inter-pressing weight variation in a number of pressing. Table 7 lists the feed time and the intra- and inter-pressing weight variations for 50 measurements. The liquid-bound granules exhibited smaller feed times and weight variations than those of the nongranulated powder. Thus, the high flowability of the granules facilitated the narrow cavity filling.

In summary, liquid-bound granules were formed by the weak bonding force called the liquid bridge force, and the bonds between their particles were easily broken by the magnetic field applied during magnetic field pressing. The utilization of these liquid-bound granules in the mass production of magnets facilitated the stable filling of small cavities with magnetic materials, thus reducing the amount of rare-earth material waste.

\section{Conclusion}

To optimize the pressing of $\mathrm{Nd}-\mathrm{Fe}-\mathrm{B}$ powder, we increased its flowability by granulation while retaining the excellent magnetic properties of this material. First, binderbound granules were prepared by the spray drying method using ethanol as the solvent and PVB as the binder. The low concentrations of PVB increased the angle of repose serving as the flowability index, while increasing the powder flowability in the binder-bound granules negatively affected their residual magnetic flux density. Thereafter, we prepared the liquid-bound granules based on the liquid bridge force. In

Table 7 Feed times and weight variations of the pressed part.

\begin{tabular}{lccc}
\hline & Feed time $(\mathrm{s})$ & Weight variation $(\%)$ \\
\cline { 3 - 4 } Non-granulated & 34 & 4.55 & 5.79 \\
Tumble granulated & 7 & 0.82 & 1.08 \\
\hline
\end{tabular}


this process, terpineol was used as the liquid due to its low volatility at room temperature and ability to be easily debind during sintering. Increasing the terpineol content in the prepared liquid-bound granules noticeably increased their flowability. A negative correlation between the flowability and residual magnetic flux density was also observed for these granules; however, they exhibited much higher residual flux densities and lower angles of repose than the binderbound granules. The liquid-bound granules containing 0.25 mass $\%$ terpineol had a repose angle of $44.2^{\circ}$ and relative residual magnetic flux density equal to $99.3 \%$ of the value obtained for the non-granulated powder. During the mass production of these granules using a narrow deep die, a high filling rate and low granule weight variations were achieved.

\section{REFERENCES}

1) M. Sagawa, S. Fujimura, H. Yamamoto, Y. Matsuura and K. Hiraga: IEEE Trans. Magn. 20 (1984) 1584-1589.

2) M. Sagawa, S. Hirosawa, H. Yamamoto and Y. Matsuura: Jpn. J. Appl. Phys. 26 (1987) 785-800.

3) M. Müller, D. Schweizer and V. Seiler: J. Bus. Ethics 138 (2016) 627648.

4) D. Brown, B.-M. Ma and Z. Chen: J. Magn. Magn. Mater. 248 (2002) $432-440$.

5) H. Tokoro and K. Uchida: IEEE Trans. Magn. 37 (2001) 2463-2466.

6) D. Hinz, A. Kirchner, D.N. Brown, B.-M. Ma and O. Gutfleisch: J. Mater. Process. Technol. 135 (2003) 358-365.

7) T. Hartwig, L. Lopes, P. Wendhausen and N. Unal: EPJ Web of Conferences 75 (2014) 04002.
8) L. Li, B. Post, V. Kunc, A.M. Elliott and M.P. Paranthaman: Scr. Mater. 135 (2017) 100-104.

9) M. Sagawa and H. Nagata: IEEE Trans. Magn. 29 (1993) 2747-2751.

10) H. Kotera, F. Tsumori, I. Iritani, T. Watanabe and S. Shima: Int. J. Powder Metallurg. 40 (2004) 33-40.

11) F.C. Franklin and L.N. Johanson: Chem. Eng. Sci. 4 (1955) 119-129.

12) O. Yamashita and Y. Kishimoto: J. Jpn. Soc. Powder Powder Metall. 43 (1996) 249-255.

13) O. Yamashita and Y. Kishimoto: J. Jpn. Soc. Powder Powder Metall. 43 (1996) 675-681.

14) Y. Kishimoto and O. Yamashita: J. Jpn. Soc. Powder Powder Metall. 44 (1997) 775-780

15) Y. Kishimoto and O. Yamashita: J. Jpn. Soc. Powder Powder Metall. 44 (1997) 951-957.

16) Y. Kishimoto and O. Yamashita: J. Jpn. Soc. Powder Powder Metall. 45 (1998) 373-378

17) K. Makita, Y. Kishimoto and O. Yamashita: J. Jpn. Soc. Powder Powder Metall. 45 (1998) 646-651.

18) R.L. Carr: Chem. Eng. 72 (1965) 163-168.

19) T. Minowa, M. Shimano and M. Honshima: J. Magn. Magn. Mater. 97 (1991) 107-111.

20) K. Hono and H. Sepehri-Amin: Scr. Mater. 67 (2012) 530-535.

21) Y. Enokido, M. Miwa, S. Goto and Y. Fujikawa: Mater. Trans. 57 (2016) 1960-1965.

22) Y. Sun, R.W. Gao, G.B. Han, G. Bai, T. Liu and B. Wang: J. Magn. Magn. Mater. 299 (2006) 82-86.

23) G. Lian, C. Thornton and M.J. Adams: J. Colloid Interface Sci. 161 (1993) 138-147.

24) Y.I. Rabinovich, M.S. Esayanur and B.M. Boudgil: Langmuir 21 (2005) 10992-10997.

25) C.E. Capes, R.J. Germain and R.D. Coleman: Ind. Eng. Chem. Process Des. Dev. 16 (1977) 517-518.

26) A. Goldszal and J. Bousquet: Powder Technol. 117 (2001) 221-231. 